psychopraxis.neuropraxis $2017 \cdot 20: 83-86$ DOI 10.1007/s00739-017-0376-8

Online publiziert: 6. Februar 2017

@ ( Der/die Autor(en) 2017. Dieser Artikel ist eine Open-Access-Publikation.

CrossMark
Markus Breu $^{1,2} \cdot$ Rainer SeidI $^{2} \cdot$ Romana Höftberger $^{1}$

${ }^{1}$ Klinisches Institut für Neurologie, Medizinische Universität Wien, Wien, Österreich

${ }^{2}$ Universitätsklinik für Kinder- und Jugendheilkunde, Medizinische Universität Wien, Wien, Österreich

\title{
Antineuronale Autoimmunenzephalitis
} Fallorientierte Darstellung

\section{Einleitung}

Über lange Zeit waren Myasthenia gravis und das Lambert-Eaton-Syndrom die einzigen bekannten Erkrankungen, deren Symptomatik durch eine reversible Autoantikörperbindung an Ionenkanäle oder Rezeptoren verursacht wird. Innerhalb des ZNS waren Antikörper vor allem in der Gruppe der paraneoplastischen neurologischen Syndrome beschrieben worden, doch deren Zielepitope sind intrazelluläre Proteine. Somit lassen sich die Symptome der betroffenen Patienten nicht durch die Antikörperbindung erklären und sind wohl am ehesten auf T-Zell-Effekte zurückzuführen. Vor etwa 10 Jahren wurde erstmals von einer immuntherapieresponsiven Form der limbischen Enzephalitis berichtet, deren Symptome durch eine reversible Antikörperbindung an Oberflächenproteine verursacht werden. Kurze Zeit später gelang durch Immunpräzipitation die Identifikation des N-Methyl-D-Aspartat-Rezeptors (NMDAR) als eines der ersten Zielepitope dieser Autoantikörper. Seitdem werden jedes Jahr ca. 1-2 neue Antikörper mit den damit assoziierten neurologischen Syndromen charakterisiert.

\section{Fallvignette}

Eine bislang gesunde 15-jährige Jugendliche wurde in einer Kinderambulanz vorstellig, da sie seit drei Nächten nicht geschlafen hatte bzw. nicht hatte schlafen können. Der Familie waren

Weitere Literatur bei den Verfassern. zusätzlich eine verwaschene Sprache, Wortfindungsstörungen und Neologismen aufgefallen. Es erfolgte die stationäre Aufnahme aufgrund ihres äußerst agitierten Verwirrtheitszustands und eines generalisierten tonisch-klonischen Krampfanfalls. Initial durchgeführte Laborparameter inklusive Toxikologie waren unauffällig. Nachdem das agitierte Zustandsbild auf mehrere Gaben Benzodiazepine (Lorazepam) nicht ansprach, erfolgte die Transferierung an eine Abteilung für Kinder- und Jugendpsychiatrie mit dem Bild einer akut psychotischen Störung mit Verwirrtheit, Angstzuständen, fahrigem und inkohärentem Duktus, fehlender Orientierung und optischen Halluzinationen. Im Akut-MRT zeigten sich mehrere max. $8 \mathrm{~mm}$ messende T2-hyperintense, diffusionseingeschräkte, KM-negative Läsionen juxtakortikal frontal und im Corpus callosum. Die psychomotorische Agitiertheit war trotz mehrfacher Lorazepam-Gaben (16 Ampullen) und Olanzapin kaum beherrschbar, weshalb die Patientin wegen weiterbestehender psychomotorischer Unruhe trotz zunehmender Somnolenz zur Überwachung an eine psychiatrische Intensivstation transferiert wurde.

Der Liquor zeigte eine leichtgradige Pleozytose, eine geringfügige Eiweißerhöhung und positive oligoklonale Banden, weshalb die Verdachtsdiagnose Enzephalitis gestellt und eine Therapie mit Zovirax und Ceftriaxon eingeleitet wurde.

Im EEG fanden sich Zeichen einer geringgradigen diffusen Hirnfunktionsstörung. Nach Ausschluss einer viralen oder bakteriellen Ursache, erfolgte unter Annahme einer neuroinflammatorischen Genese eine 5-tägige Kortison-Stoßtherapie mit anschließendem PrednisolonTapering sowie eine zweitägige IgG-Gabe ( $2 \mathrm{~g} / \mathrm{kgKG}$ ). Trotz dieser Therapie entwickelte die Patientin ein katatones $\mathrm{Zu}$ standsbild mit massiv dyston erhöhtem Musekeltonus, starrem Blick, intermittierenden choreatiformen Bewegungen der Arme und Beine und intermittierender bizzarer dystoner Handhaltung. Der Nachweis von Antikörpern gegen den NMDA-Rezeptor gelang im Serum und Liquor und bestätigte die Diagnose einer NMDAR-Enzephalitis.

Ein Tumorscreening mittels MRT von Thorax und Abdomen ergab keinen Hinweis auf Teratome. Unter der weiteren Therapie mit insgesamt 11 Plasmaseparationen, mehreren IgG-Gaben und zweimaliger Verabreichung von Rituximab kam es über die folgenden vier Wochen zu einer deutlichen Besserung und Aufklärung.

Annähernd zwei Monate nach Erstmanifestation konnte die Patientin unter oraler Therapie mit Cellcept, Prednisolon und 6-wöchigen IgG-Gaben entlassen werden. Klinisch zeigte die Patientin zu diesem Zeitpunkt eine völlige Remission, konnte sich an die Geschehnisse der letzten Wochen jedoch nicht erinnern.

\section{NMDAR-Enzephalitis}

Die NMDAR-Enzephalitis wurde 2007 als erstes Krankheitsbild aus der Gruppe der antineuronalen antikörperassoziierten Enzephalitiden beschrieben [1]. Unter allen Ursachen einer Enzephali- 
Tab. 1 ZNS-Erkrankungen mit Antikörpern gegen neuronale synaptische oder Oberflächenantigene (adaptiert nach [2])

\begin{tabular}{|c|c|c|}
\hline Antigen & Hauptsyndrom & Haupterstmanifestation \\
\hline NMDAR & NMDAR-Enzephalitis & $\begin{array}{l}\text { Psychose, Krampfanfälle, Bewegungsstö- } \\
\text { rung (Kinder) }\end{array}$ \\
\hline AMPAR & Limbische Enzephalitis & Gedächtnisstörung, Verwirrung \\
\hline $\mathrm{GABA}_{B} \mathrm{R}$ & Limbische Enzephalitis & Gedächtnisstörung, Krampfanfälle \\
\hline LGI1 & Limbische Enzephalitis & Gedächtnisstörung, Krampfanfälle \\
\hline CASPR2 & $\begin{array}{l}\text { Morvan-Syndrom, limbische Enze- } \\
\text { phalitis }\end{array}$ & $\begin{array}{l}\text { Gedächtnisstörung, Schlafstörung, Neuro- } \\
\text { myotonie }\end{array}$ \\
\hline $\mathrm{GABA}_{A} \mathrm{R}$ & $\begin{array}{l}\text { Enzephalitis mit kortikal-subkorti- } \\
\text { kalen MRT-FLAIR-Veränderungen }\end{array}$ & Krampfanfälle \\
\hline DPPX & Enzephalitis & Diarrhoe, Gewichtsverlust, Hyperekplexie \\
\hline Dopamin2R & Basalganglienenzephalitis & Parkinsonismus \\
\hline mGluR5 & Enzephalitis & Gedächtnisstörung, Verwirrung \\
\hline Neurexin-3a & Enzephalitis & Verwirrung, Krampfanfälle \\
\hline $\operatorname{lgLON} 5$ & $\begin{array}{l}\text { Non-REM und REM-Schlafstörung, } \\
\text { Hirnstammdysfunktion }\end{array}$ & Schlafstörung \\
\hline DNER (Tr) & Zerebelläre Ataxie & Gangstörung \\
\hline $\begin{array}{l}\text { P/Q type } \\
\text { VGCC }\end{array}$ & Zerebelläre Ataxie & Gangstörung \\
\hline mGluR1 & Zerebelläre Ataxie & Gangstörung \\
\hline GlyR & PERM, Stiff-Person-Syndrom & Rigidität, Spasmen \\
\hline
\end{tabular}

tis ist sie häufiger als jede einzelne virale Entität und gleichzeitig die häufigste Autoimmunenzephalitis. In einer großen Kohorte wurde ein Altersspektrum von 8 Monaten bis 85 Jahren bei Erstmanifestation beschrieben. Der Altersmedian lag bei 21 Jahren.

Der NMDA-Rezeptor gehört zur Gruppe der glutamatergen Rezeptoren und spielt eine essenzielle Rolle bei der Langzeitpotenzierung, dem Gedächtnis und der Kognition. In Zellkulturen und einem Mausmodell mit kontinuierlicher Liquorinfusion von Antikörpern konnte gezeigt werden, dass NMDAR-Antikörper zu einer dosisabhängigen, reversiblen Internalisierung von NMDA-Rezeptoren, erhöhten Glutamatausschüttung und erhöhten kortikomotorischen Erregbarkeit führen. Sobald die Antikörper durch die Immuntherapie entfernt werden, kommt es zu einer Wiederherstellung der normalen Rezeptorverhältnisse. Es wird angenommen, dass es zu einer rein funktionellen Einschränkung der synaptischen Transmission kommt, im Gegensatz zu einer z. B. irreversiblen T-Zellmediierten Neuronenschädigung.

Die klinischen Symptome einer Blockade des Rezeptors sind größtenteils bekannt durch die Anwendung NMDAR- blockierender Substanzen in der Anästhesie (z. B. Ketamin) und durch illegalen Drogenkonsum (z. B. Phencyclidin, „Angel Dust"), die zu illusionärer Perzeption, paranoiden Gedanken und Defiziten exekutiver Funktionen führen. Diese Symptome entsprechen den milderen Symptomen einer NMDAR-Enzephalitis, die sich generell in Form einer stadienhaften Progression entwickelt. Oftmals beginnt die Erkrankung mit milden Prodromalsymptomen, die einer banalen viralen Infektion gleichen. Einige Tage bis Wochen später kommt es klassisch zu zunehmenden Einschränkungen durch eine Kombination psychiatrischer (Psychose, Agitiertheit und Katatonie) und neurologischer Symptome (Krampfanfälle, Vigilanzstörung, Bewegungsstörungen und autonome Dysfunktion). Diese können im zeitlichen Verlauf weiter progredient sein und bis zu Koma und Tod führen. In den meisten Fällen kommt es unter Therapie zu einer stadienhaften Rückbildung der Symptome in annähernd umgekehrter Reihenfolge des Auftretens. Die Art der Symptome ist weitgehend abhängig vom Alter des Patienten, wobei sich generell Kinder (je jünger umso mehr) eher mit neurologischen Symptomen wie Krampfanfällen und Bewegungsstörun-
Tab. 2 Diagnostische Kriterien für Auto-

immunenzephalitis (adaptiert nach [3])

Für eine Diagnose müssen alle $\mathbf{3}$ Kriterien erfüllt sein:

1. Subakuter Beginn (rasche Progression, $<3$ Monate) von

a. Arbeitsgedächtnisstörung (Kurzzeitgedächtnis) oder

b. veränderter mentaler Zustand (Vigilanz, Lethargie, Persönlichkeit) oder

c. Psychiatrische Symptome

2. Zumindest 1 der Folgenden:

a. Neue fokale neurologische Symptome

b. Krampfanfälle (nicht erklärbar durch Epilepsiesyndrom)

c. Liquorpleozytose

d. MRT-Läsionen (FLAIR-Hyperintensitäten in einem oder beiden medialen Temporallappen [limbische Enzephalitis]) oder multifokale Areale in grauer und/oder weißer Substanz kompatibel mit Demyelinisierung oder Entzündung

3. Ausschluss anderer Ursachen

gen präsentieren (50\%), während Erwachsene eher Verhaltensauffälligkeiten zeigen (65\%).

I) Die NMDAR-Enzephalitis ist häufig mit ovariellen Teratomen assoziiert

Des Weiteren konnten bei etwa $8 \%$ der Patientin mit akuter Psychose NMDARAntikörper detektiert werden. Der behandelnde Arzt sollte sich nicht auf einzelne Untersuchungsmethoden verlassen, da diese auch negativ sein können (MRT in $67 \%$, EEG in $10 \%$, Liquor in $21 \%$ unauffällig).

Die Entdeckung der Erkrankung erfolgte u. a. aufgrund ihrer Assoziation mit Teratomen. In etwa $40 \%$ der NMDAREnzephalitis-Patienten wird ein Tumor gefunden. In $94 \%$ der Fälle handelt es sich dabei um ovarielle Teratome, die NMDAR-positive Neurone beinhalten. Die frühe und konsequente Tumorsuche ist von hoher klinischer Relevanz, da eine Entfernung mit einem besseren klinischen Ergebnis assoziiert ist.

Therapeutisch wird zwischen Firstline- und Second-line-Immuntherapie unterschieden. Als „first-line“ gelten Ste- 
roide, intravenöse Immunglobuline und Plasmapheresen. Second-line-Therapien beinhalten Rituximab, Cyclophosphamid und andere Immunsuppressiva. Da gezeigt werden konnte, dass eine rasch eskalierende Immuntherapie mit einem besseren Ergebnis assoziiert ist, entschließen sich mittlerweile viele behandelnde Ärzte dazu, möglichst rasch mit einer Kombinationstherapie aus Rituximab und Cyclphosphamid zu beginnen.

Generell hat die NMDAR-Enzephalitis trotz ihrer oftmals sehr schwerwiegenden Präsentation eine relativ gute Prognose. In etwa $75 \%$ der Fälle kommt es zu einer kompletten Remission, auch wenn diese oft erst nach mehreren Monaten erreicht wird. Rückfälle treten in ca. $12 \%$ der Fälle auf und sind meist mit einer unzureichenden langfristigen Immuntherapie assoziiert.

\section{Weitere antineuronale Antikörper}

Seit der Erstbeschreibung der NMDAREnzephalitis wurden bis dato (Stand Januar 2017) eine Reihe weiterer antineuronaler antikörperassoziierter Erkrankungen beschrieben (- Tab. 1). Die Symptome sind vielfältig und reichen u. a. von Gedächtnisstörungen (z.B. NMDAR, AMPAR, GABAbR) über Schlafstörungen (IgLON5) bis hin zu Parkinsonismus (D2R) und Stiff-Person-Syndrom (GlycinR, Amphiphysin). Neben der NMDAR-Enzephalitis sind in der psychiatrischen Differenzialdiagnose besonders Antikörper gegen AMPAR und $\mathrm{GABA}_{\mathrm{B}} \mathrm{R}$ von Relevanz.

\section{AMPA-Rezeptor-Enzephalitis}

Antikörper gegen den glutamatergen AMPA-Rezeptor führen $\mathrm{zu}$ einer Erkrankung, die sich klassisch als limbische Enzephalitis mit subakuten Verwirrtheitszuständen, Orientierungs- und Gedächtnisstörung manifestiert. Ein Teil der Patienten zeigt auch Krampfanfälle. In der Mehrzahl der Fälle lassen sich eine Liquorpleozytose und T2/FLAIR-positive MRT-Läsionen, überwiegend im Temporallappen lokalisiert, nachweisen. Die Erkrankung ist häufig mit Tumoren von Lunge, Brust und Thymus assoziiert. Auch wenn die Mehrzahl an Patienten auf eine Immuntherapie anspricht, kommt es oftmals zu Rückfällen.

\section{$\mathrm{GABA}_{\mathrm{B}}$-Rezeptor-Enzephalitis}

Der $\mathrm{GABA}_{\mathrm{B}}$-Rezeptor wird prä- und postsynaptisch exprimiert und dient der Hyperpolarisation der Nervenzelle am Ende des Aktionspotenzials. Antikörper gegen die B1-Untereinheit des Rezeptors führen zu einer limbischen Enzephalitis mit charakteristischen schweren Krampfanfällen bis hin zu einem Status epilepticus. In etwa der Hälfte der betroffenen Patienten findet sich ein Bronchialkarzinom (SCLC). Die Mehrzahl der Patienten spricht gut auf eine Immuntherapie an.

\section{Diagnose}

Die hochgradige Verdachtsdiagnose einer „Autoimmunenzephalitis“ kann gestellt werden, wenn die in - Tab. 2 angeführten Kriterien erfüllt sind. Liquor und Serum dieser Patienten sollte möglichst rasch einem spezialisierten Labor zugeführt werden. Die entsprechenden Abnahmen sollten möglichst frühzeitig bzw. in der Phase der stärksten klinischen Symptomatik durchgeführt werden, da die Schwere der Symptomatik oft direkt mit dem Antikörpertiter korreliert. Von Bedeutung ist, dass sowohl Liquor als auch Serum untersucht werden, da ein fehlender Nachweis von Antikörpern in einer der Proben antineuronale Antikörper in der anderen nicht ausschließt.

Im Falle der NMDAR-Enzephalitis hat Liquor eine höhere Sensitivität als Serum (100\% vs. $85 \%)$.

Für den Nachweis antineuronaler Oberflächenrezeptorantikörper stehen dem Labor im Wesentlichen zwei Testmethoden zur Verfügung. In der einen Methode erfolgt der Nachweis mittels zellbasiertem Assay („cell-based assay“, CBA). Hierfür werden humane embryonale Nierenzellen (HEK-Zellen) mit einem Plasmid transfiziert, was dazu führt, dass die Zellen ein bestimmtes Antigen an der Oberfläche exprimieren. Eine Doppelfärbung mit einem konventionellen Antikörper des entsprechenden psychopraxis.neuropraxis $2017 \cdot 20: 83-86$ DOI 10.1007/s00739-017-0376-8

(c) Der/die Autor(en) 2017. Dieser Artikel ist eine Open-Access-Publikation.

M. Breu · R. Seidl · R. Höftberger

Antineuronale

Autoimmunenzephalitis. Fallorientierte Darstellung

\section{Zusammenfassung}

Vor etwa 10 Jahren wurde eine neue Kategorie von Autoimmunenzephalitiden entdeckt, die durch Autoantikörper gegen synaptische oder Oberflächenantigene verursacht werden und in der psychiatrischen Praxis von hoher Relevanz sind. Insbesondere bei subakuter Psychose, Gedächtnisstörungen und einer Kombination psychiatrischer und neurologischer Symptome sollten die Autoimmunenzephalitiden in die Differenzialdiagnose eingeschlossen werden. Mit der Testung von Liquor und/oder Serum in einem spezialisierten Labor können die bekannten Antikörper innerhalb weniger Tage detektiert werden. Eine frühe Diagnose und rasche Immuntherapie führen in den meisten Fällen zu einer Remission der Symptome.

\section{Schlüsselwörter}

Autoimmunenzephalitis · Autoimmunenzephalitiden · Antikörper · Immuntherapie · Psychiatrie

\section{Antineuronal autoimmune encephalitis. Case-oriented presentation}

\section{Abstract}

Approximately 10 years ago, a new category of autoimmune encephalitis was described that is caused by autoantibodies against synaptic or surface antigens and has a high relevance for psychiatric care. In particular, in cases of subacute psychosis, memory deficit, and combination of neurological and psychiatric symptoms, the differential diagnoses should include autoimmune encephalitis. In a specialized lab, the known antibodies can be detected in cerebrospinal fluid and/or serum within a couple of days. Early diagnosis and immunotherapy are crucial for a favorable outcome and lead in most cases to complete remission.

\section{Keywords}

Autoimmune encephalitis . Autoimmune disease · Antibodies - Immunotherapy . Psychiatry 


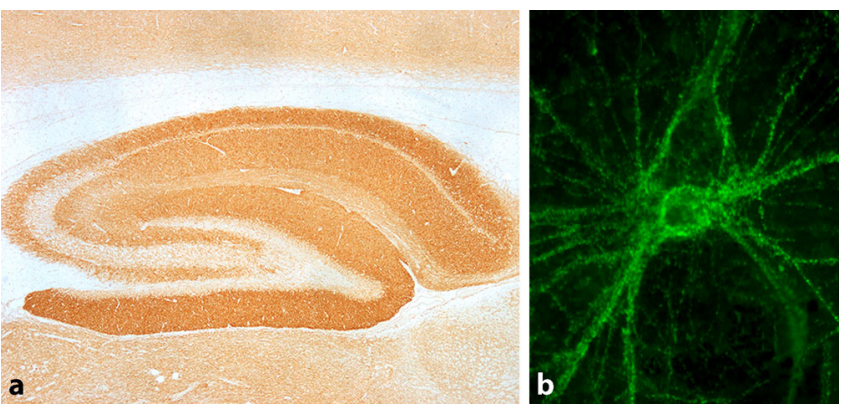

Abb. 1 a a Der Liquor eines Patienten mit NMDAR-Enzephalitis zeigt im gewebebasierten Assay im Hippocampus ein kräftiges Neuropilfärbemuster. b Der Liquor eines Patienten mit bislang uncharakterisierten antineuronalen Oberflächenrezeptorantikörpern zeigt eine starke Membranfärbung einer lebenden kultivierten Nervenzelle. @ Romana Höftberger

Antigens und der Patientenprobe bestätigt das Vorhandensein des Antikörpers in der Probe. Eine andere Methode umfasst das Screening mittels gewebebasiertem Assay („tissue-based assay“, TBA). Hierbei wird die Patientenprobe (Liquor und/oder Serum) auf einem Gehirnschnitt einer gesunden Ratte oder Maus inkubiert. Bei vorhandenen Antikörpern färbt sich das Gewebe mittels Sekundärantikörper und einer chemischen Reaktion (DAB-Reaktion) in charakteristischer Weise an (• Abb. 1a). Diese Methode hat den Vorteil, dass nicht nur bekannte Antikörper detektiert werden können, sondern eventuell auch bisher nicht identifizierte. Neue Antikörper können in einem weiteren Schritt in Forschungslabors mittels Färbung lebender Nervenzellkulturen (• Abb. 1b) und anschließender Immunpräzipitation charakterisiert werden.

\section{Ausblick}

Bei etwa $30 \%$ der Patienten, deren klinische Präsentation und Therapieansprechen hochgradig auf eine antikörpermediierte Ursache hinweisen, lässt sich keiner der bisher identifizierten antineuronalen Antikörper nachweisen. Diese Beobachtung legt nahe, dass es ein breites Feld an bisher unbekannten antikörperassoziierten Erkrankungen gibt. Insbesondere aufgrund der hohen prognostischen Bedeutung einer frühen Immuntherapie sollte diese Krankheitsgruppe möglichst frühzeitig in die Differenzialdiagnose eingeschlossen werden und gegebenenfalls auch ohne unmittelbaren
Nachweis von Antikörpern empirisch behandelt werden.

\section{Fazit für die Praxis}

- Autoimmunenzephalitiden werden durch Antikörper gegen synaptische oder Oberflächenantigene auf Neuronen hervorgerufen.

- Die Erstmanifestation beinhaltet häufig psychiatrische Symptome im Sinne subakuter psychotischer Störungen.

- Die häufigste Form ist die NMDAREnzephalitis mit einer klassischen Kombination aus Agitiertheit, Gedächtnisstörung, Vigilanzminderung und Epilepsie.

- Die NMDAR-Enzephalitis ist häufig mit ovariellen Teratomen assoziiert.

- Eine Bestätigung der Diagnose erfolgt durch den direkten Nachweis von Antikörpern in Liquor und/oder Serum in einem spezialisierten Labor.

- Die Therapie beinhaltet immuntherapeutische Ansätze mit Kortison, IVIG (IgG), Plasmapherese/ Immunadsorption, Rituximab oder Cyclophosphamid.

- Bei frühzeitiger Immuntherapie und Tumorresektion kommt es in den meisten Fällen zu einer kompletten Remission.

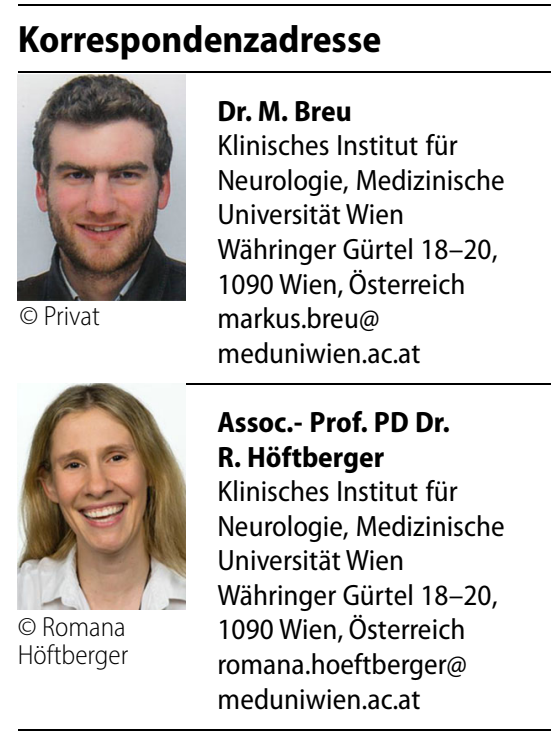

Open access funding provided by Medical University of Vienna.

\section{Einhaltung ethischer Richtlinien}

Interessenkonflikt. M. Breu, R. Seidl und R. Höftberger geben an, dass kein Interessenkonflikt besteht.

Dieser Beitrag beinhaltet keine von den Autoren durchgeführten Studien an Menschen oder Tieren.

Open Access. Dieser Artikel wird unter der Creative Commons Namensnennung 4.0 International Lizenz (http://creativecommons.org/licenses/by/4.0/deed. de) veröffentlicht, welche die Nutzung, Vervielfältigung, Bearbeitung, Verbreitung und Wiedergabe in jeglichem Medium und Format erlaubt, sofern Sie den/die ursprünglichen Autor(en) und die Quelle ordnungsgemäßnennen, einen Link zur Creative Commons Lizenz beifügen und angeben, ob Änderungen vorgenommen wurden.

\section{Literatur}

1. Dalmau, J., Tüzün, E., Wu, H., Masjuan, J., Rossi, J.E., Voloschin, A., Baehring, J.M., et al. (2007). Paraneoplastic anti-N-methyl-D-aspartate Receptor Encephalitis Associated with Ovarian Teratoma. Annals of Neurology 61(1): 25-36. doi:10.1002/ana.21050

2. Dalmau,J.(2016). NMDA ReceptorEncephalitis and Other Antibody-mediated Disorders of the Synapse: The 2016 Cotzias Lecture. Neurology 87(23): 2471-82. doi:10.1212/WNL.0000000000003414

3. Graus, F., Titulaer, M. J., Balu, R., Benseler, S., Bien, C. G., Cellucci, T., Cortese, I., et al. (2016). A Clinical Approach to Diagnosis of Autoimmune Encephalitis. The Lancet. Neurology 15(4):391-404. doi:10.1016/S1474-4422(15)00401-9 